\title{
Colonisation d'un nouvel habitat par Culex pipiens (Diptera, Culicidae) : le creux d'arbre des subéraies en pays Zaer, Maroc
}

\author{
G. Metge' \\ N. Belakoul2
}

Mots clés: Diptera, Culicidae, écologie, taxonornie, Culex pipiens, habitat, Maroc.

Les conditions de sécheresse qui ont sévi au Maroc de 1981 à 1983 ont favorisé la sélection d'un nouvel habitat par Culex pipiens. espèce particulièrement plastique, le creux d'arbre en suberaie. La description morphologique de la larve du stade 4 , de la nymphe et des imagos $\sigma$ et $\$$ mont re que ces populations dendrolimniques s'apparentent à la forme Culex pipiens autogenicus.

Colonisation of a new habitat by Culex pipiens (Diptera : Culicidae) : hollows in the cork-layer of trees in the Zaer district, Morocco.

Keywords : Diptera, Culicidae, ecology, taxonomy, Culex pipiens, habitat, Morocco.

The dry conditions which occurred in Morocco from 1981 to 1983 have resulted in the selection of a new habital by Culex pipients, which is a very adaptable species, in hollows of trees. Morphological descriptions of the fourth larval instar, the pupae and the male and female adults have shown that these populations living in trec holes (phytotelmata) belong to the form $C$. pipiens autogenicus.

\section{Introduction}

Au cours des années 1983-1984, les pêches larvaires dans les creux d'arbres des suberaies de Ben Slimane, Sidi Bettache (fig 1) de la Meseta occidentale marocaine, nous ont révélé la présence inhabituelle d'une espèce, Culex pipiens $L$.

Cette dernière, jusqu'à ce jour, n'a été signalée que par Seguy (1921, in Rioux 1958) et Gau (1953) comme accidentelle des creux d'arbres.

Nos prospections régulières ont permis de vérifier la constance saisonnière de Culex pipiens, de 1983 à 1984, dans cet habitat ; la colonisation intervient en avril et se poursuit jusqu'à l'assèchement juin-juillet, pour ure densité assez élevée de l'ordre de 100 à 300 larves par cavité.

\footnotetext{
1. Institut National d'Etudes Supérieures de Biologie, BP 358. Tlemcen. Algérie.

2. Ecole Normale Supérieure, Casablanca, Maroc.
}

\section{Situation écologique de l'habitat}

Les suberaies de la Meseta occidentale marocaine (Ben Slimane, Sidi Bet tache) se situent en ambiance, semi-aride à hiver temperé (Sauvage 1961, Metge 1986). Leur développement résulte d'une compensation de facteurs liée à la présence d'une nappe phréa. tique proche de la surface et alimentée par de petites dépresssions endoréiques à caractere temporaire (dayas). Sur le talus périphérique de ces cuvettes s'installent les individus de chêne liège.

L'exploitation du liège par l'homme contribue à l'entretien de ces forêts, notamment l'élagage pour favoriser la croissance d'un fùt principal. La trace qu'il en résulte, généralement à la base de l'arbre, se transforme progressivement sous l'action des micro-organismes, en cavités de 1 à 5 litres de volume, dont l'orifice présente une surface de quel. ques centimètres carrés à $\mathbf{1}$ ou $\mathbf{2}$ décimètres carrés. 


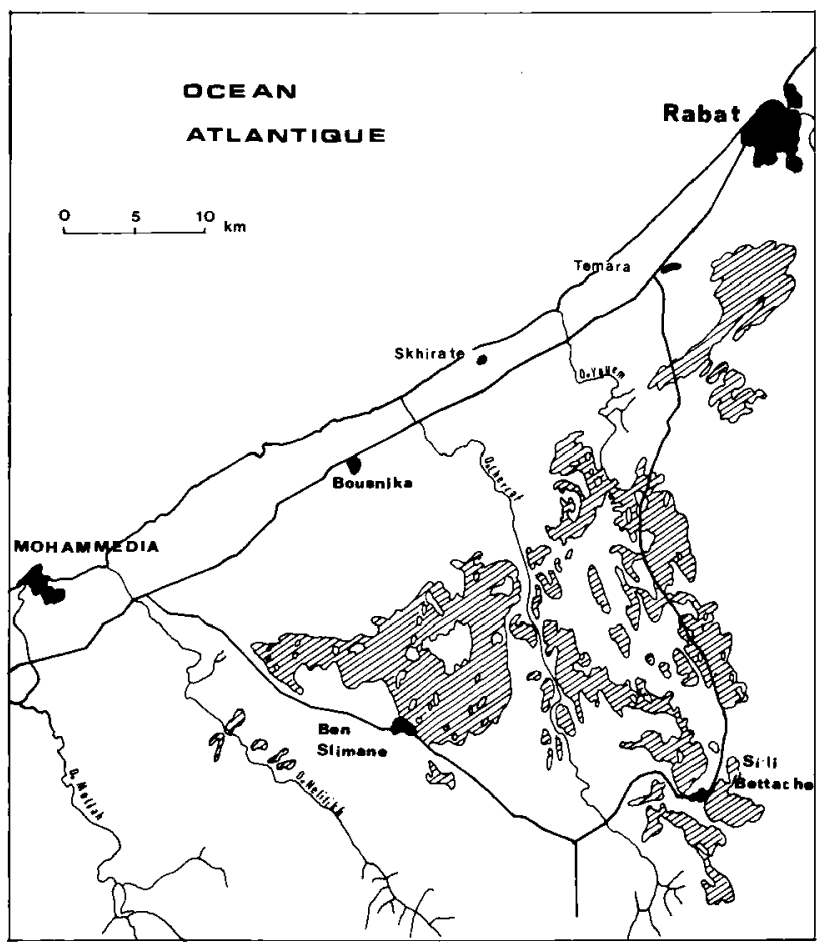

Fig. 1. Localisation géographique des milieux étudiés.

Deux facteurs contribuent à la mise en eau de ces creux :

Les précipitations et l'écoulement gravitaire des nappes perchées temporaires qui peuvent les ali. menter localement par transit souterrain.

\section{Influence du climat sur la sélection d'un nouvel habitat par Culex pipiens}

La situation climatique présentée sur la figure 2, par les diagrammes ombrothermiques, met en évidence deux périodes:
- une normale, correspondant aux données météorologiques de 1933 à 1983 :

- l'autre, detaillée de 1981 à 1984. En période normale, 5 mois de sècheresse centrés sur la période chaude (juin, juillet, août, septembre, octobre) le cycle biologique de Culex pipiens se déroule comme suit :

- reprise d'activité des imagos en mars, avril:

- pontes et développement complet des stades immatures en avril, mai et souvent juin sur les plans d'eau résiduels des dayas. 

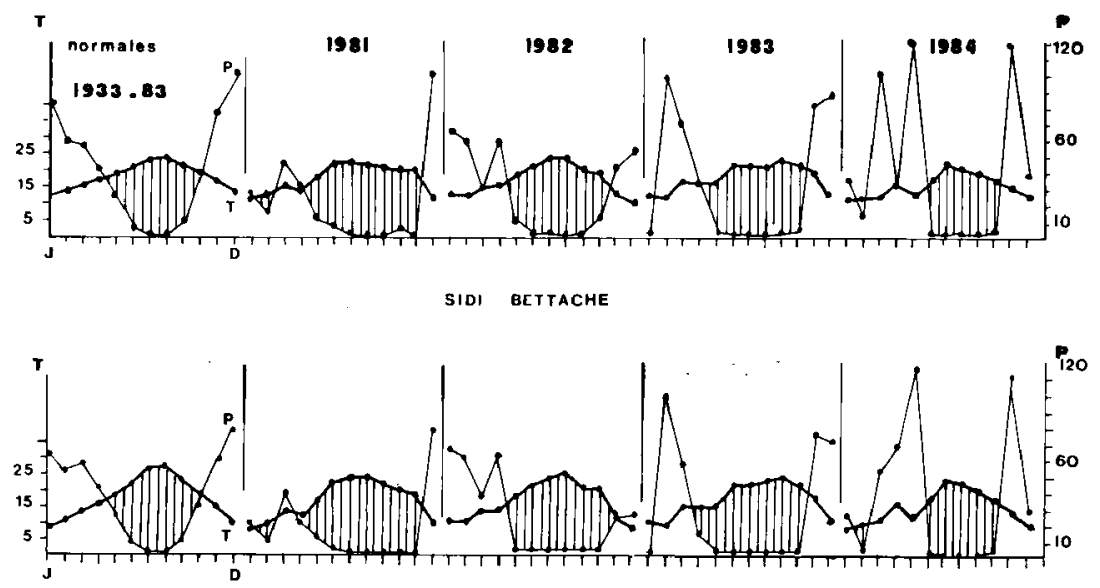

Fig. 2. Diagrammes ombrothermiques

Parallèlement les creux d'arbres sont colonisés par des espèces typiquement arboricoles, Aedes echinits, A. geniculatus, A. berlandi etc... (Metge \& EJ Alaoui 1987).

Nos observations de 1980 confirment cette ségrégation des espèces liée à deux types d'habitats fondamentalement différents : le creux d'arbre et la daya (Belakoul 1985).

De 1981 a fin 1983 intervient une longue période de sècheresse qui s'étale sur 9 à 10 mois par an.

En 1983, nous découvrons pour la première fois C. pipiens dans les creux d'arbres, des le mois de mars jusqu'à l'assec, début juin (fig. 3).

Par contre, la présence de $C$. pipiens dans les dayas, pour la même période devient éphémère. En effet, la reprise de l'activité des imagos en mars avril correspond à l'assec des mares temporaires : seules quelques petites flaques résiduelles permettent le développement pré-imaginal sur un laps de temps de quelques jours.

Au vu de ces résultats, il apparaît clairement que la sècheresse prolongée joue un rôle de barrière

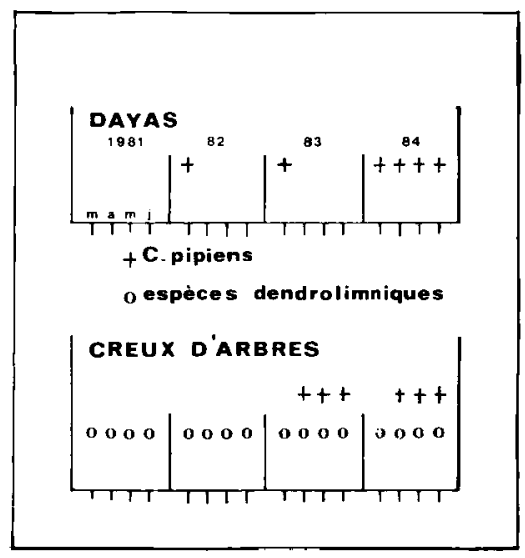

Fig. 3. Distribution spatio-temporelle des Culicides 
interdisant ou réduisant considérablement le développement de $C$ pipiens de 1981 à 1983 dans les dayas.

Les creux d'arbres de la suberaie restent les seuls points d'eau disponibles aux femelles de $C$. pipiens pour déposer leurs ceufs et permettre leur développement. Deux années auront été nécessaires avant que cette sélection puisse s'opérer.

Notons enfin, qu'en 1984, les conditions météorologiques étant revenues normales $(5$ mois de sècheresse) les populations de $C$. pipiens se sont développées normalement dans les dayas et les creux d'arbres.

\section{Description morphologique des popula- tions dendrolimniques de Culex pipiens}

Les descriptions des larves du $4^{e}$ stade, de l'hypopygium mâle, de la nymphe, portent sur un effectif de 50 individus, les caracteres retenus correspondent à $80 \%$ des individus observés.

\section{LARVE DU 4c STADE (fig. 4 a,b,d)}

- Le siphon.

Généralement sub-cylindrique, assez mince, porte quatre touffes des soies irrégulièrement insérées. Le nombre de branches est variable ( 2 à 5 ). Les dents du peigne siphonique, d'un nombre moyen de 14 (12 à 18) sont régulières. Le siphon présente une longueur moyenne de $1,36 \mathrm{~mm}$. Les crochets siphoniques sub-apicaux sont grêles, longs et presque droits, les apicaux sont grêles, longs et presque droits, les apicaux sont sombres, courts et droits.

\section{- L'abdomen.}

Les dents du VIII ${ }^{e}$ segment, régulières, à barbules apicales offrent un port réduit, leur nombre varie de 40 à 50 .

\section{L'IMAGo MÃLE}

Le rapport trompe/palpe est en moyenne 0,7.

- L'hypopygium (fig. 5 a,b,c)

Le coxite est large, couvert de soies sombres, fortes et courtes. Le lobe un peu avant le 1/4 apical du coxite se divise en deux parties dont la proximale est plus développée. Cette demière porte 3 appendices forts, droits, courbés à l'extrémité ; un autre appendice fort se trouve en bas du lobe. La deuxième partie du lobe porte trois autres appendices courts et courbés à l'apex. Le style large à la base, mince à l'apex se courbe progressivement. Les deuxièmes plaques du mésosome ne touchent pas aux parmèes et sont réduites à la courbure apicale.

\section{L'IMAGO FEMELLE}

Il ne présente pas de caractères sensiblement différents au standard $C$. pipiens décrits par Rioux (1958), Harbach et al (1985).

\section{La NYMPHE (fig. $6 a, b)$}

Les trompettes respiratoires présentent un pavillon dont l'ouverture s'inscrit dans un rectangle, sa dimension correspond au $1 / 3$ de la trompette.

* Les nageoires, larges dans leur partie médiane et apicale, présentent une échancrure nette et deux épines apicales dont l'une est bifurquée.

\section{LE CHÉtogRAMME}

Utilisé par de nombreux auteurs pour étudier le complexe pipiens, le chétogramme s'obtient en utilisant :

- A : l'index siphonique (L/l) rapport de la longueur du siphon à la largeur (l);

- les quatre touffes de soies siphoniques (B,C,D,E):

B est le nombre moyen de branches de la touffe la plus basale:

C celui de la deuxième touffe en partant de la base :

D celui de la troisième touffe :

E celui de la touffe apicale ; d'après Marschall et al. (1951) in Senevet \& Andarelli (1959).

Callot, in Rioux (1958) ajoute la valeur Mt (divisée par 2 pour la commodité de la représentation graphique) qui correspond au nombre moyen des dents au mentum, non compris la dent centrale.

Les résultats consignés sur la figure 7 montrent que la forme dendromét rique de $C$. pipiens s'apparente à $C$. pipiens autogenicus.

Ces données viennent conforter nos observations sur la forme hypopygiale, qui elle aussi s'apparente à $C$. pipiens autogenicus. 


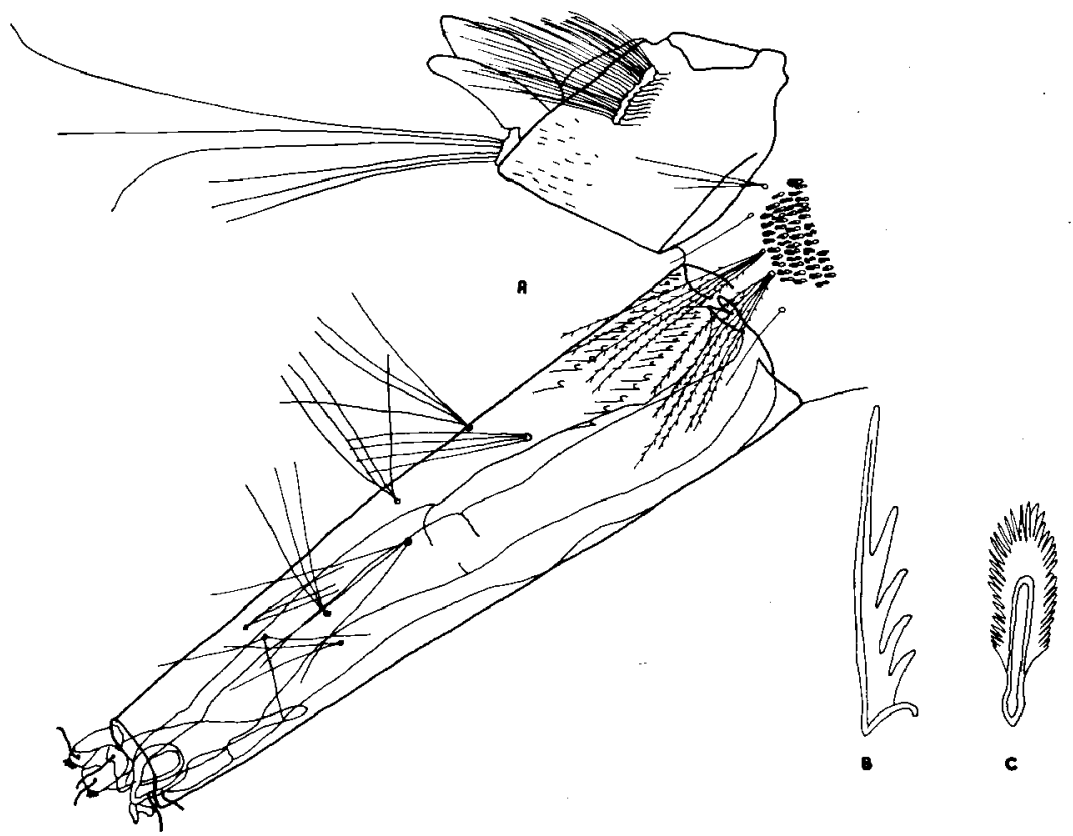

Fig. 4. A : siphon et VIII segment d'une larve de $4^{e}$ stade $; \mathbf{B}:$ dent du peigne siphonique ; $\mathrm{C}$ : dent du peigne du viII segment. 

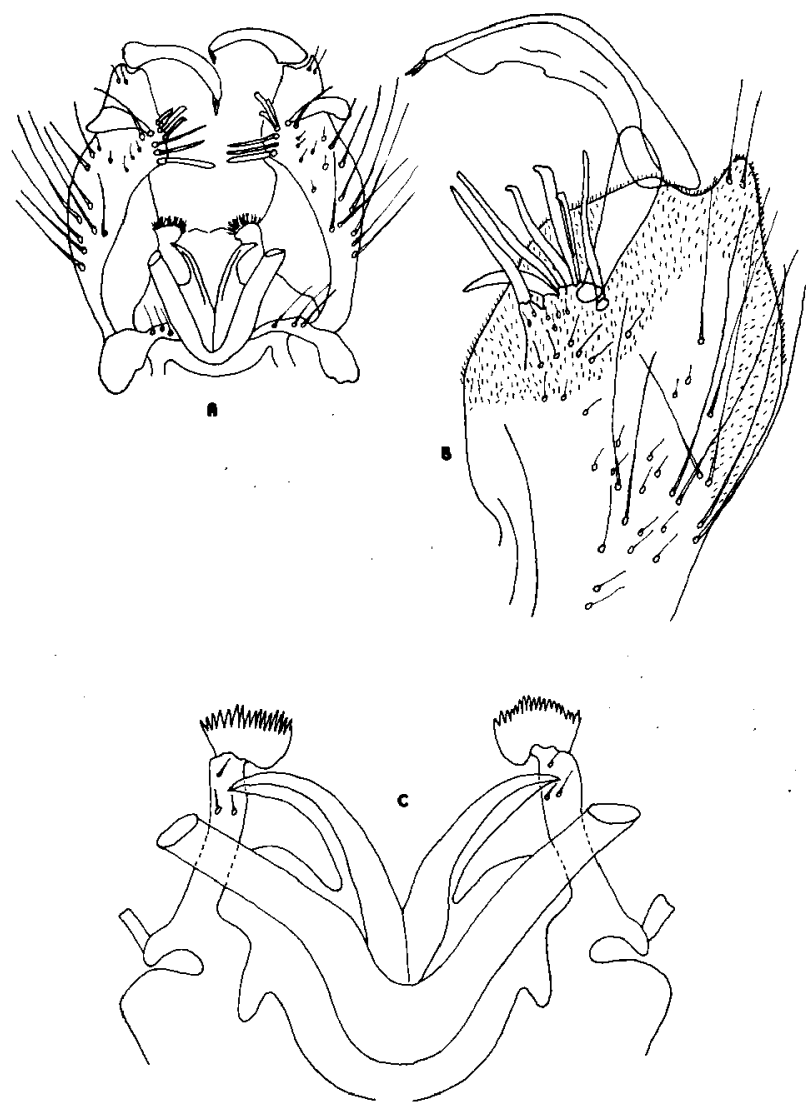

Fig. $5 . \mathrm{A}$ : vue générale de l'hypopygium; $\mathrm{B}$ : coxite lobe apical et style : $\mathrm{C}$ : plaques du mésosome et paramère. 


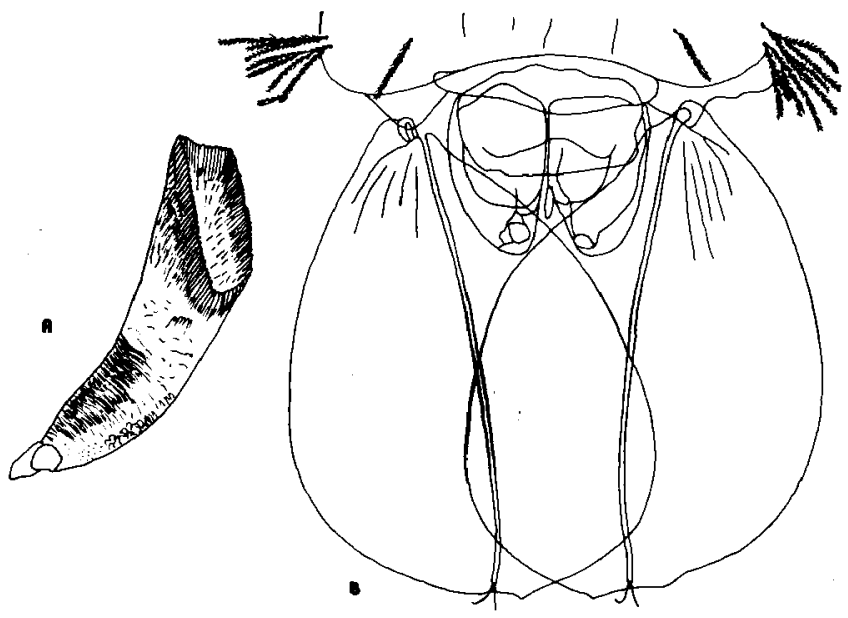

Fig. 6. Détails morphologiques de la nymphe. A : trompette; B : nageoires

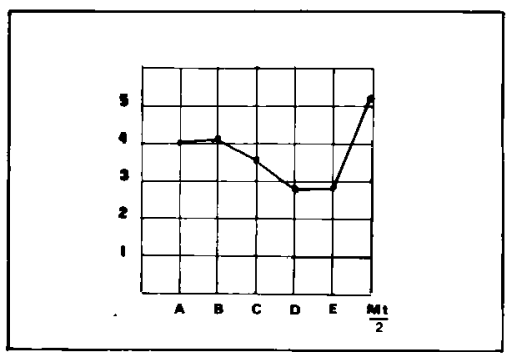

Fig. 7. Chétogramme : C. pipiens du creux d'arbre : valeurs moyennes sur 50 individus 


\section{Conclusion}

L'opportunité de nos recherches dans un contexte climatique exceptionnel pour le Maroc, nous a permis de mettre en relief le facteur déterminant (sècheresse prolongée) qui favorise la plasticité phénotypique de certaines populations dans la colonisation d'un nouvel habitat.

Les descriptions morphologiques mettent en évidence un certain nombre de particularités au niveau de l'hypopyge, du siphon et du mentum qui apparentent ces populations dendrolimiques à la forme C. pipiens autogenicus.

\section{Travaux cités}

Belakoul (N.). 1985. - Contribution à l'étude de la faune culicidienne dendrolimnique de la suberaie en pays Zaer (Maroc). Colonisation par Culex pipiens d'un nouvel habitat : causalité et étude comparće de deux formes de Culex pipiens D.E.A. Univers. P. Sabatier Toulouse, 1-113.
Gaud (J.). 1953. - Notes biogéographiques sur les Culicides du Maroc. Inst. Pasteur, Maroc, IV (7): 443-490.

Harbach (R.E.), Dahl (C.) \& White (G.B.). 1985. - Culex pipiens Lin nacus (Diptera : Culicidae) concepts type designations, and description. Proceedings of the Entomol. Society of Washington. 87 (1): 1-24.

Metge (G.). 1986. - Etude des écosystèmes hydronorphes (daya el merja) de la Meseta occidentale marocaine. Typologie et synthèse cartographique a objectif sanitaire appliquèe aux populations d'Anopheles labranchiae (Falleroni, 1926) Diptera, Culicidae. Anophelinae). Thèse d'état ès Sciences, AixMarseille : $280 \mathrm{p}$,

Metge (G.) \& El Alaoui (M.). 1987. - Euude de la dynamique des populations d'Aedes echinus (Culicide dendrolimnique) en écophase aquatique au Maroc. Annls Limnol., 23 (2): 129.134.

Rioux (J A.), 1958. - Les Culicides du * Midi * méditerranéen. Enc. ent. XXX, P. Lechevalier, Paris, 1-303.

Sauvage (Ch.). 1961. - Recherches géobotaniques sur les suberaies marocaines. Trav. Insi. Sci, chérif., sér. bot., 21 : 1462.

Senevet (G.) \& Andarelli (L.). 1959. - Les moustiques de l'A frique du nord et du bassin méditerranéen. Enc. ent. XXXVII, P. Lechevalier, Paris : $383 \mathrm{p}$. 